\title{
VARIATIONS ON A HYPERGEOMETRIC THEME
}

\author{
Michael MilgRAM
}

Abstract. The question was asked: Is it possible to express the function

$$
h(a) \equiv{ }_{4} F_{3}(a, a, a, a ; 2 a, a+1, a+1 ; 1)
$$

in closed form [1]? After considerable analysis, the answer appears to be "no", but during the attempt to answer this question, a number of interesting (and unexpected) related results were obtained, either as specialized transformations, or as closed-form expressions for several related functions. The purpose of this paper is to record and review both the methods attempted and the related identities obtained, the former for their educational merit, the latter because they do not appear to exist in the literature. Specifically, new ${ }_{4} F_{3}(1),{ }_{5} F_{6}(1)$ and generalized Euler sums (those containing digamma functions) are presented along with a detailed discussion of the methods used to obtain them.

Mathematics subject classification (2010): 33-02, 33B15, 33C20,33E20.

Keywords and phrases: 3F2(1), 4F3(1),5F6(1), hypergeometric, transformations, digamma, summation.

\section{REFERENCES}

[1] Dalimil Mazac and Miguel F. Paulos, Analytic functionals for the 1d conformal bootstrap, 2018, to be published.

[2] A. R. Miller AND R. B. PARIS, Clausen's series ${ }_{3} F_{2}(1)$ with integral parameter differences and transformations of the hypergeometric function ${ }_{2} F_{2}(x)$, Integral Transforms and Special Functions $\mathbf{2 3}$ (1): 21-33, 2012.

[3] A. P. Prudnikov, Yu. A. Brychkov, and O. I. Marichev, Integrals and Series: More Special functions, vol. 3, Gordon and Breach Science Publishers, New York, 1986.

[4] L. J. Slater, Generalized Hypergeometric Functions, Cambridge University Press, 1966.

[5] Eldon R. Hansen, A Table of Series and Products, Prentice-Hall Inc., Englewood Cliffs, N. J., 1975.

[6] R. W. Gosper, Decision procedure for indefinite hypergeometric summation, Proc. Nat. Acad. of Sciences of the USA, 75 (1), 1978, also available from https://www.ncbi.nlm.nih.gov/pmc/ articles/PMC411178/.

[7] W. N. BAILEy, Generalized hypergeometric series, Cambridge tracts in mathematics and mathematical physics, The University Press, 1935.

[8] JACQUES RAYNAL, On the definition and properties of generalized 6j symbols, Journal of Mathematical Physics 20 (12): 2398-2415, 1979.

[9] Allen R. Miller, A transformation formula for the generalized ${ }_{4} F_{3}(1)$, J. Phys. A: Math. Gen. 36: 11853-11858, 2003.

[10] CHOi J. RATHIE A. AND P. CHOPRA, A new proof of the extended Saalschütz's summation theorem for the series ${ }_{4} F_{3}$ and its application, Honam Mathematical Journal 35 (3): 407-415, 2013.

[11] B. M. Minton, Generalized hypergeometric function of unit argument, J. Math. Phys. 11 (4): 1375$6,1970$.

[12] P. W. KARLSSON, Hypergeometric functions with integral parameter differences, J. Math. Phys. 12 (2): 270-271, 1971.

[13] H. M. SRIVASTAVA, Generalized hypergeometric functions with integral parameter differences, Indagationes Mathematicae (Proceedings), 76 (1): 38-40, 1973. 
[14] J. E. GotTschalk And E. N. MASLEn, Reduction formulae for generalised hypergeometric functions of one variable, Journal of Physics A: Mathematical and General 21 (9): 1983, 1988.

[15] M. SAIGO AND R. K. SAXENA, Expansions of ${ }_{4} F_{3}$ when the upper parameters differ by integers, ArXiv Mathematics e-prints, March 1998, https://arxiv.org/abs/math/9803165.

[16] Wenchang CHU, Analytic formulae for extended 3F2 - series of Watson-Whipple-Dixon with two extra integer parameters, Mathematics of Computation 81: 467-479, 2012.

[17] M. S. Milgram, 447 instances of hypergeometric 3F2(1), ArXiv e-prints, May 2011, https://arxiv.org/abs/1105.3126; also https://www.researchgate.net/publication/ 51891949.

[18] M. A. Shpot And H. M. SRIVAStaVA, The Clausenian hypergeometric function ${ }_{3} F_{2}$ with unit argument and negative integral parameter differences, Applied Mathematics and Computation 259: 819-827, May 2015, also available from ArXiv e-prints,1411.2455v3, April 15, 2015, doi/10.1016/j.amc.2015.03.031.

[19] M. MÜller AND D. SCHLEICHER, How to add a noninteger number of terms: From axioms to new identities, American Mathematical Monthly 118 (2): 136-152, 2011, also available from https://arxiv.org/abs/1001.4695.

[20] H. Wilf And D. Zeilberger, Rational functions certify combinatorial identities, J. Amer. Math. Soc. 3: 147-158, 1990, available on request via https : //www.researchgate.net/publication/ 2447078.

[21] Y. LUKE, The special functions and their approximations, Volume I, volume 53 of Mathematics in Science and Engineering, Academic Press, 1969.

[22] C. Krattenthaler, Rao K. Srinivasa, On Group Theoretical Aspects, Hypergeometric Transformations and Symmetries of Angular Momentum Coefficients, in: Symmetries in Science XI, edited by Gruber B. J., Marmo G., Yoshinaga N., Springer, 2004, available from http://www . mat. univie.ac.at/ Kratt/artikel/bregenz1.html.

[23] A. EBISU AND K. IWAS AKI, Three-Term Relations for 3F2(1), ArXiv e-prints, 1604.00480, Apr 2016.

[24] Ranjan Roy George E. Andrews, Richard Askey, Special Functions, Volume 71 of Encyclopedia of Mathematics and its Applications, Cambridge University Press, Cambridge, England, 2000.

[25] Ranjan Roy, Binomial identities and hypergeometric series, The American Mathematical Monthly 94 (1): 36-46, 1987, also available from: https : //carma.newcastle.edu.au/jon/Preprints/ Books/MbyE/Second-Ed/Material/3F2/roy-maa.pdf.

[26] W. F. ShEPPARD, Summation of the coefficients of some terminating hypergeometric series, Proc. London Math. Soc. s2-10 (1): 469-478, 1912.

[27] E. S. ANDERSEN, Two summation formulae for products sums of binomial coefficients, Math. Scand. 1: 261-262, 1953.

[28] Michael Milgram, On some sums of digamma and polygamma functions - revised 2017, 2017, available from https://arxiv.org/abs/math/0406338 or https://www.researchgate.net/ publication/320371548.

\section{APPENDICES}

\section{A. 4-part Transformations of a ${ }_{4} F_{3}(1)$}

\section{A.1. Miller's transforms}

Miller [9, Eqs. (1.1) and (1.2)] has obtained the following two 4-part transformations among arbitrary ${ }_{4} F_{3}(1)$.

$$
\begin{aligned}
& { }_{4} F_{3}(a, b, c, d ; e, f, g ; 1) \\
= & \Gamma(e) \Gamma(f) \Gamma(g) \Gamma(1-d) \\
& \times\left(\frac{\Gamma(b-a) \Gamma(c-a){ }_{4} F_{3}(a, 1+a-e, 1+a-f, 1+a-g ; 1+a-d, 1+a-b, 1+a-c ; 1)}{\Gamma(b) \Gamma(c) \Gamma(e-a) \Gamma(f-a) \Gamma(g-a) \Gamma(1+a-d)}\right.
\end{aligned}
$$




$$
\begin{aligned}
& +\frac{\Gamma(a-b) \Gamma(c-b)_{4} F_{3}(b, 1+b-e, 1+b-f, 1+b-g ; 1+b-d, 1+b-a, 1+b-c ; 1)}{\Gamma(a) \Gamma(c) \Gamma(e-b) \Gamma(f-b) \Gamma(g-b) \Gamma(1+b-d)} \\
& \left.+\frac{\Gamma(a-c) \Gamma(b-c){ }_{4} F_{3}(c, 1+c-e, 1+c-f, 1+c-g ; 1+c-d, 1+c-a, 1+c-b ; 1)}{\Gamma(b) \Gamma(a) \Gamma(e-c) \Gamma(f-c) \Gamma(g-c) \Gamma(1+c-d)}\right)
\end{aligned}
$$

and

$$
\begin{aligned}
& { }_{4} F_{3}(a, b, c, d ; e, f, g ; 1) \\
= & \frac{\Gamma(e) \Gamma(f) \Gamma(1-d) \Gamma(1-c)}{\Gamma(1-g)} \\
& \times\left(\frac{\Gamma(a-b) \Gamma(1+b-g)_{4} F_{3}(b, 1+b-g, 1+b-f, 1+b-e ; 1+b-c, 1+b-d, 1+b-a ; 1)}{\Gamma(a) \Gamma(e-b) \Gamma(f-b) \Gamma(1+b-c) \Gamma(1+b-d)}\right. \\
& +\frac{\Gamma(b-a) \Gamma(1+a-g)_{4} F_{3}(a, 1+a-g, 1+a-e, 1+a-f ; 1+a-c, 1+a-d, 1+a-b ; 1)}{\Gamma(b) \Gamma(e-a) \Gamma(f-a) \Gamma(1+a-c) \Gamma(1+a-d)} \\
& \left.-\frac{\Gamma(g-1) \Gamma(1+a-g) \Gamma(1+b-g){ }_{4} F_{3}(1+b-g, 1+a-g, 1+c-g, 1+d-g ; 2-g, 1+e-g, 1+f-g ; 1)}{\Gamma(a) \Gamma(b) \Gamma(g-c) \Gamma(g-d) \Gamma(1+e-g) \Gamma(1+f-g)}\right)
\end{aligned}
$$

\section{A.2. Meijer G-function transforms}

Any hypergeometric function ${ }_{p} F_{q}\left(a_{p} ; b_{q} ; z\right)$ can always be written as a Meijer Gfunction [21, Chapter V] and the reverse. The original intent of the Meijer G-function was to assign meaning to ${ }_{p} F_{q}(z)$ when $p>q+1$ by the magic of contour integration and the residue theorem. This is summarized by the general result [21, Eq. 5.3(1)]

$$
G_{p, q}^{m, n}\left(z \mid \begin{array}{l}
a_{p} \\
b_{q}
\end{array}\right)=G_{q, p}^{n, m}\left(\frac{1}{z} \mid \begin{array}{l}
1-b_{q} \\
1-a_{p}
\end{array}\right) \quad \arg \frac{1}{z}=-\arg z .
$$

which shows that the interchange of $a_{p} \leftrightarrow 1-a_{p}$ and $b_{q} \leftrightarrow 1-b_{q}$ together with $p \leftrightarrow q$ is equivalent to the analytic continuation $z \rightarrow 1 / z$ for any ( $m$ and $n$ combination(s) of) ${ }_{p} F_{q-1}(z)$. Of course when $p=q$ and $z=1$ this is equivalent to a transformation among (combinations of) ${ }_{q} F_{q-1}(1)$. For example, with $z=1$ in (A.3) along with the prescription [21, Eq. 5.2(7)] for expanding a G-function into a combination of ${ }_{p} F_{q-1}(1)$, and using $(-1)^{a}=\exp (-i \pi a)$ as a template for dealing with terms that arise in the expansion, rewrite both sides in the form of (a) combination(s) of ${ }_{4} F_{3}(1)$ by setting $m=1, n=4, p=q=4$. The imaginary part of the resulting expression must vanish, because for the moment, without loss of generality, it is permissible to require that the variables $a, \ldots g \in \mathbb{R}$. Solving that equation yields an apparently hitherto unrecognized 4-part transformation as follows

$$
\begin{aligned}
& { }_{4} F_{3}(a, b, c, d ; e, f, g ; 1) \\
= & -\frac{\Gamma(1-a) \Gamma(1-b) \Gamma(1-d) \Gamma(1-c)}{\Gamma(1-f) \Gamma(1-e) \Gamma(1-g)}
\end{aligned}
$$




$$
\begin{aligned}
& \times\left(\frac{\Gamma(g-1) \Gamma(g-f) \Gamma(g-e){ }_{4} F_{3}(1-g+a, 1-g+b, 1-g+c, 1-g+d ; 2-g, f+1-g, e+1-g ; 1)}{\Gamma(g-b) \Gamma(g-c) \Gamma(g-d) \Gamma(g-a)}\right. \\
& +\frac{\Gamma(e-1) \Gamma(e-f) \Gamma(e-g)_{4} F_{3}(1-e+a, 1-e+b, 1-e+c, 1-e+d ; 2-e, f+1-e, g+1-e ; 1)}{\Gamma(e-b) \Gamma(e-c) \Gamma(e-d) \Gamma(e-a)} \\
& \left.+\frac{\Gamma(f-1) \Gamma(f-e) \Gamma(f-g)_{4} F_{3}(1-f+a, 1-f+b, 1-f+c, 1-f+d ; 2-f, e+1-f, g+1-f ; 1)}{\Gamma(f-b) \Gamma(f-c) \Gamma(f-d) \Gamma(f-a)}\right)
\end{aligned}
$$

whose validity can be expanded to include the variables $a, \ldots g \in \mathbb{C}$ because of the principle of analytic continuation (parameter space is analytic). This relationship appears to be independent of (A.1) and (A.2). However, it is easily shown that the transformation arising from the real part of that same expression is a symmetric permutation of, and therefore equivalent to, (A.1). It is an open question which of the many other permutations of variables among (A.1), (A.2) and (A.4) will result in new, independent transformations applicable to ${ }_{4} F_{3}(1)$, analogous to Whipple's categorization of threepart transformations for hypergeometric ${ }_{3} F_{2}(1)$ discussed in [21, Section 3.13]. See Appendix C. This is discussed at some length in [8] and [22]. 


\section{B. Application to a ${ }_{3} F_{2}(1)$}

In Section ??, the motivation for considering transformations among ${ }_{3} F_{2}(1)$ was presented. With this in mind, several relationships involving special cases of ${ }_{3} F_{2}(1)$ are (re)derived and collected in this Appendix.

\section{B.1. Special cases of Miller, Paris, Shpot and Srivastava}

A simple relevant transformation can be obtained using Miller and Paris [2, Eq. (1.7)] where it is shown for $m>n$ that

$$
\begin{aligned}
& { }_{3} F_{2}(a, b, n ; c, m ; 1) \\
= & \frac{\Gamma(m) \Gamma(1-a) \Gamma(1-b)}{\Gamma(n) \Gamma(1-c)} \sum_{k=0}^{m-n-1} \frac{(-1)^{k} \Gamma(n+k) \Gamma(1-c+n+k)}{\Gamma(k+1) \Gamma(m-n-k) \Gamma(1-a+n+k) \Gamma(1-b+n+k)} \\
+ & \frac{\Gamma(m) \Gamma(c) \Gamma(1-a) \Gamma(1-b)}{\Gamma(c-a) \Gamma(c-b) \Gamma(m-n)} \sum_{k=0}^{n-1} \frac{(-1)^{k} \Gamma(k+m-n) \Gamma(c-a-b+k+m-n)}{\Gamma(k+1) \Gamma(n-k) \Gamma(1-a+k+m-n) \Gamma(1-b+k+m-n)} .
\end{aligned}
$$

Formally, generalize (B.1) by extending the upper limit of both sums to infinity (they are truncated by the denominator Gamma functions if $m, n \in \mathbb{N}$ ) straightforwardly identifying each of the extended sums as a hypergeometric function. Then relax the above condition by generalizing such that $m, n \in \mathbb{R}$, to obtain

$$
\begin{aligned}
& { }_{3} F_{2}(a, b, c ; e, f ; 1) \\
= & \frac{\Gamma(f) \Gamma(1-a) \Gamma(1-b) \Gamma(-e+c+1)_{3} F_{2}(c,-f+c+1,-e+c+1 ; 1-b+c, 1-a+c ; 1)}{\Gamma(1-e) \Gamma(f-c) \Gamma(1-a+c) \Gamma(1-b+c)} \\
& +\frac{\Gamma(f) \Gamma(e) \Gamma(1-a) \Gamma(1-b) \Gamma(e-a-b+f-c)}{\Gamma(e-a) \Gamma(e-b) \Gamma(c) \Gamma(1-a+f-c) \Gamma(1-b+f-c)} \\
& \times{ }_{3} F_{2}(f-c, 1-c, e-a-b+f-c ; 1-b+f-c, 1-a+f-c ; 1),
\end{aligned}
$$

a known result (see (C.20) below) that resides in [21, Tables (3.3), (3.4) and(3.5)], valid for all values of the parameters $a, b, c, e, f$. This observation justifies the apparently $a d$ hoc replacement $c \rightarrow e, m \rightarrow f, n \rightarrow c$ in (B.1) to yield (B.2).

REMARK. Reverse this procedure by setting $f \rightarrow m$ and $c \rightarrow n$ to obtain a much simpler derivation of (B.1) from the known result (B.2).

Similarly, Shpot and Srivastava [18] have obtained a result for a more general related problem as follows:

$$
\begin{aligned}
& { }_{3} \mathrm{~F}_{2}(a, b, c ; b+1+m, c+1+n ; z) \\
= & \frac{\Gamma(b+1+m) \Gamma(c+1+n)}{\Gamma(b) \Gamma(c)} \sum_{i=0}^{m} \sum_{j=0}^{n} \frac{(-1)^{i}(-1)^{j}}{\Gamma(1-i+m) \Gamma(n+1-j) \Gamma(i+1) \Gamma(j+1)} \\
& \times\left(\frac{{ }_{2} \mathrm{~F}_{1}(a, c+j ; c+j+1 ; z)}{(c+j)(b-c+i-j)}+\frac{{ }_{2} \mathrm{~F}_{1}(a, b+i ; b+i+1 ; z)}{(b+i)(c-b+j-i)}\right)
\end{aligned}
$$


After a somewhat lengthy analysis, in the case that $z=1$, they reduce this result to a 3-part transformation [18, Eq. (31)] (see (D.11)) among terminating ${ }_{3} F_{2}(1)$ on the right, and the same ${ }_{3} F_{2}(1)$ that appears in (B.3) on the left. However, following the same logic as outlined above, by setting

$$
\begin{aligned}
& m \rightarrow e-1-b \\
& n \rightarrow f-1-c
\end{aligned}
$$

we obtain a generalization of Shpot and Srivastava's result [18, Eq. (31)] (see (D.11)) in the form of a well known 3-part transformation, specifically [21, Eq. 3.13.3(11)], and explicitly

$$
\begin{aligned}
& { }_{3} \mathrm{~F}_{2}(a, b, c ; e, f ; 1) \\
= & \Gamma(1-a) \Gamma(f) \Gamma(e)\left(\frac{\Gamma(c-b){ }_{3} \mathrm{~F}_{2}(b,-e+1+b, b+1-f ; b-c+1, b+1-a ; 1)}{\Gamma(c) \Gamma(e-b) \Gamma(b+1-a) \Gamma(-b+f)}\right. \\
& \left.+\frac{\Gamma(b-c){ }_{3} \mathrm{~F}_{2}(c,-f+c+1,-e+c+1 ; c+1-a, c-b+1 ; 1)}{\Gamma(b) \Gamma(c+1-a) \Gamma(e-c) \Gamma(f-c)}\right) .
\end{aligned}
$$

REMARK 1. Reversing (B.4), gives a simple derivation of [18, Eq. (31)] from the known result (B.5).

REMARK 2 AND DigRESSION. In (B.3), set $z=1$, sum (and reverse) the various series that arise and compare with [18, Eq. (31)] to find an interesting contiguity relation (also see [23]):

$$
{ }_{3} \mathrm{~F}_{2}(c,-n, b ; a, b+1+m ; 1)=\frac{(-1)^{m} \Gamma(1-b)}{\Gamma(-b-m)} \sum_{i=0}^{m} \frac{(-1)^{i}{ }_{3} \mathrm{~F}_{2}(c,-n, b+i ; a, b+i+1 ; 1)}{(-b-i) \Gamma(i+1) \Gamma(1-i+m)} .
$$

\section{B.2. Obvious reduction from a ${ }_{4} F_{3}$}

Set a pair of the top and bottom parameters in (A.1) to be equal (e.g. $a=e$ ), and thereby trivially produce the three-part transformations among ${ }_{3} F_{2}(1)$ reproduced above (B.5).

With a similar choice of reduction $(e=a)$ and reassignment of variable names in (A.4), another three-part transformation among ${ }_{3} F_{2}(1)$ results. This result is equivalent to applying (A.3) in the case $m=1, n=1, p=q=3$. Although this transformation involves terms clearly embedded in [21, Tables 3.3, 3.4 and 3.5] it is given explicitly below:

$$
\begin{aligned}
& { }_{3} \mathrm{~F}_{2}(a, b, c ; f, e ; 1) \\
= & -\frac{\Gamma(1-b) \Gamma(1-c) \Gamma(1-a)}{\Gamma(1-f) \Gamma(1-e)} \\
& \times\left(\frac{\Gamma(-1+f) \Gamma(-e+f)_{3} \mathrm{~F}_{2}(1+a-f, 1+b-f, 1+c-f ; 2-f, 1+e-f ; 1)}{\Gamma(f-a) \Gamma(-b+f) \Gamma(f-c)}\right. \\
& \left.+\frac{\Gamma(-1+e) \Gamma(e-f){ }_{3} \mathrm{~F}_{2}(1+a-e, 1+b-e, 1+c-e ; 2-e, 1+f-e ; 1)}{\Gamma(e-a) \Gamma(e-b) \Gamma(e-c)}\right) .
\end{aligned}
$$


Because of the asymmetry among the parameters of the ${ }_{4} F_{3}$ appearing in (A.2), various reductions from a ${ }_{4} F_{3}$ to a ${ }_{3} F_{2}$ can be made by choosing to equate different combinations of top and bottom parameters to yield different transformations. For example, the case $g=d$ produces a symmetric permutation equivalent to (B.5) whereas choosing $e=a$ and reassigning variables generates the following mixture, all of which are included (see Appendix C) in [21, Tables (3.3), (3.4) and (3.5)]:

$$
\begin{aligned}
{ }_{3} \mathrm{~F}_{2}(a, b, c ; e, f ; 1)= & \Gamma(1+b-e) \Gamma(1-a) \Gamma(1-c) \Gamma(f) \\
& \times\left(\frac{{ }_{3} \mathrm{~F}_{2}(b, 1+b-e, 1+b-f ; 1+b-c, 1+b-a ; 1)}{\Gamma(1-e) \Gamma(f-b) \Gamma(1+b-c) \Gamma(1+b-a)}\right. \\
& \left.+\Gamma(e) \frac{{ }_{3} \mathrm{~F}_{2}(1+b-e, 1+a-e, 1+c-e ; 2-e, 1-e+f ; 1)}{\Gamma(2-e) \Gamma(-c+e) \Gamma(e-a) \Gamma(1-e+f) \Gamma(b)}\right) .
\end{aligned}
$$

\section{B.3. Curious reduction from a ${ }_{4} F_{3}(1)$}

A curious combination arises by setting $g=d$ in (A.1) to obtain

$$
\begin{aligned}
& { }_{3} \mathrm{~F}_{2}(a, b, c ; e, f ; 1) \\
= & \frac{\Gamma(e) \Gamma(f)}{\sin (\pi d)} \\
& \times\left(-\frac{\sin (\pi(a-d))_{3} \mathrm{~F}_{2}(a, 1+a-e, 1+a-f ; 1+a-c, 1+a-b ; 1) \Gamma(c-a) \Gamma(b-a)}{\Gamma(f-a) \Gamma(e-a) \Gamma(c) \Gamma(b)}\right. \\
& -\frac{\Gamma(a-b) \sin (\pi(b-d))_{3} \mathrm{~F}_{2}(b, 1+b-e, 1+b-f ; 1+b-a, 1+b-c ; 1) \Gamma(c-b)}{\Gamma(a) \Gamma(c) \Gamma(f-b) \Gamma(e-b)} \\
& \left.-\frac{\Gamma(a-c) \Gamma(b-c) \sin (\pi(-d+c)){ }_{3} \mathrm{~F}_{2}(c, 1+c-e, 1+c-f ; 1+c-a, 1+c-b ; 1)}{\Gamma(a) \Gamma(f-c) \Gamma(e-c) \Gamma(b)}\right) .
\end{aligned}
$$

Notice the appearance of the arbitrary parameter $d$ on the right-hand side but not on the left. Various possibilities were studied for various choices of the parameter $d$; even the choice $d=0$ yielded nothing other than one of the results quoted above. A typical choice of $d=1 / 2$ yields nothing more than a four-part transformation among ${ }_{3} F_{2}(1)$ functions (but also see (C.5)). However, a second even more curious transformation exists: set $g=a$ in (A.2) to obtain another transformation, again with a superflous and arbitrary parameter $a$ appearing on the right-hand side, but not the left, this time embedded within the parameters of the hypergeometric functions themselves, rather than in a multiplicative factor. That is, after redefining parameters (including $a:=d$ ):

$$
\begin{aligned}
& { }_{3} \mathrm{~F}_{2}(a, b, c ; e, f ; 1) \\
= & \frac{\Gamma(1+b-d) \Gamma(1-c) \Gamma(1-a) \Gamma(f) \Gamma(e)}{\Gamma(1-d)} \\
& \times\left(\frac{{ }_{3} \mathrm{~F}_{2}(b, 1+b-e, 1+b-f ; 1+b-a, 1+b-c ; 1) \Gamma(d-b)}{\Gamma(d) \Gamma(1+b-a) \Gamma(1+b-c) \Gamma(f-b) \Gamma(e-b)}\right. \\
& -\frac{\Gamma(d-b){ }_{4} \mathrm{~F}_{3}(1, d, 1-e+d, 1-f+d ; 1+d-a, 1+d-b, 1+d-c ; 1)}{\Gamma(f-d) \Gamma(e-d) \Gamma(1+d-b) \Gamma(1+d-c) \Gamma(1+d-a) \Gamma(b)}
\end{aligned}
$$




$$
\left.-\frac{\Gamma(d-1){ }_{4} \mathrm{~F}_{3}(1,1+a-d, 1+b-d, 1+c-d ; 2-d, 1+e-d, 1+f-d ; 1)}{\Gamma(d) \Gamma(d-a) \Gamma(d-c) \Gamma(1+e-d) \Gamma(1+f-d) \Gamma(b)}\right)
$$

Although the case $d=0$ appears to be superficially interesting, after simplification, the result yields nothing more profound than the simple and well known reduction

$$
{ }_{4} \mathrm{~F}_{3}(1, a, b, c ; 2, e, f ; 1)=\frac{(e-1)(f-1)\left({ }_{3} \mathrm{~F}_{2}(a-1, b-1, c-1 ; e-1, f-1 ; 1)-1\right)}{(a-1)(b-1)(c-1)}
$$

The result (B.10) however offers potential for investigating the properties of sums involving digamma functions, because the left hand-side vanishes under the action of the operator $\frac{\partial}{\partial d}$.

\section{Appendix: A primer on 3-part relations among ${ }_{3} F_{2}(1)$}

The following should be read in conjunction with Section 3.13.3 of Luke's book [21]; Slater's book [4, Section 4.3.2] also covers much of the same material.

The three part relations among ${ }_{3} F_{2}(1)$ were classified by Whipple (1923), summarized by Bailey [7, Section 3.5] and reproduced by Luke [21, Section 3.13.3] and Slater [4, Section 4.3.2]. To say that the notation is obscure would be an understatement, and perhaps that is why these relations are often overlooked in the literature (e.g. see (B.2) and (B.5)). The following is an addendum to, and clarification of, Whipple's classification, the basis of which is a set of six parameters $r_{i}$ where $i=0(1) 5$. To the best of my knowledge, these have never been explicitly listed in the literature. Working backwards from [21, Table 3.3] it is possible to obtain these parameters in terms of the top and bottom parameters of a canonical function ${ }_{3} F_{2}(a, b, c ; e, f ; 1)$ and thereby clarify the underlying algorithm. In summary,

$$
\begin{aligned}
& r_{0}=5 / 6+c / 3+b / 3-2 / 3 e-2 / 3 f+a / 3 \\
& r_{1}=-2 / 3 b+e / 3-1 / 6+f / 3+a / 3-2 / 3 c \\
& r_{2}=f / 3-2 / 3 a+e / 3-1 / 6+b / 3-2 / 3 c \\
& r_{3}=c / 3-1 / 6+e / 3-2 / 3 b+f / 3-2 / 3 a \\
& r_{4}=e / 3-1 / 6+b / 3-2 / 3 f+a / 3+c / 3 \\
& r_{5}=-1 / 6+b / 3-2 / 3 e+f / 3+a / 3+c / 3,
\end{aligned}
$$

from which, with the help of [21, Eq. (3.13.3(13)] it is possible to reproduce [21, Table 3.3] in its entirety, and calculate representative (i.e. mixed combination of $a, b, c, e, f$ ) top and bottom parameters $\alpha_{l m n}$ and $\beta_{m n}$ respectively (see [21, Eq. 3.13(13)]), labelled by distinct permutations of integers $l, m, n=0(1) 5$. Whipple then introduces two fundamental functions $F_{p}(u ; v, w)$ and $F_{n}(u ; v, w)$ [21, Eqs. 3.13.3(14) and 3.13.3(15)] defined in terms of ${ }_{3} F_{2}(a, b, c ; e, f ; 1)$, where each of the labels $u, v, w$ take on one of the distinct, but different, numbers $0,1 \ldots 5$. Representative independent mixtures of the top and bottom parameters are classified and listed in [21, Tables 3.4 and 3.5]; any missing combinations from the tables represent simple, irrelevant permutations among the top three, or between the bottom two, parameters of the canonical ${ }_{3} F_{2}(1)$. The 
important point to note is that only the first parameter of $F_{p}(u ; v, w)$ or $F_{n}(u ; v, w)$ is important; the second and third parameters represent different mixtures among the top and bottom parameters and are conveniently omitted unless needed for clarity or specificity. Because of the two-part (Thomae) relations, all twenty of these functions with the same first parameter are equal; that is

$$
F_{p}\left(u ; v_{1}, w_{1}\right)=F_{p}\left(u ; v_{2}, w_{2}\right)
$$

and

$$
F_{n}\left(u ; v_{1}, w_{1}\right)=F_{n}\left(u ; v_{2}, w_{2}\right)
$$

for any permitted combination of $u, v, w=0(1) 5$ (no duplication). In the following, it is assumed that the arguments of any function on either side of an equality sign are such that the series representation converges (if $s \equiv e+f-a-b-c$, then $\mathfrak{R}(s)>$ 0 ); otherwise, the equality between sides must be interpreted in the sense of analytic continuation. With this notation, [21, Eq. 3.13.3(11)] (also [3, Eq. 7.4.4(3)] or (B.5)), a well known three-part relation among three particular ${ }_{3} F_{2}(1)$, can be written in labelled form

$$
F_{p}(0 ; 4,5)=\frac{\pi \Gamma\left(\alpha_{023}\right)}{\sin \left(\pi \beta_{23}\right)}\left(\frac{F_{n}(2)}{\Gamma\left(\alpha_{134}\right) \Gamma\left(\alpha_{135}\right) \Gamma\left(\alpha_{345}\right)}-\frac{F_{n}(3)}{\Gamma\left(\alpha_{124}\right) \Gamma\left(\alpha_{125}\right) \Gamma\left(\alpha_{245}\right)},\right)
$$

or, in expanded form

$$
\begin{aligned}
F_{p}(0 ; 4,5)= & -\Gamma(c+1-b) \Gamma(b-c) \Gamma(1-a) \\
& \times\left(\frac{F_{n}(2 ; 3,1)}{\Gamma(-b+e) \Gamma(-b+f) \Gamma(c)}-\frac{F_{n}(3 ; 1,2)}{\Gamma(e-c) \Gamma(f-c) \Gamma(b)}\right) .
\end{aligned}
$$

In (C.5), an arbitrary choice of (superfluous) second and third parameters of $F_{n}(2)$ and $F_{n}(3)$ have been included, and the arguments of the $\Gamma$ functions have been written explicitly in terms of the underlying parameters, to specify one of the 20 possibilities equivalent to (B.5). By limiting the left-hand side to one particular (the canonical) ${ }_{3} F_{2}(1)$, and removing the second and third parameters from the right-hand side, (C.4) represents a family of forty 3-part relations among different combinations of ${ }_{3} F_{2}(1)$ selected by different combinations of second and third parameters (see [21, Table 3.13.3.5]) on the right-hand side. Three other (equivalent families of) three-part relations among ${ }_{3} F_{2}(1)$ are known and listed in [3, Eqs. 7.4.4(4)-(6)]. The first two of these, once parsed according to the tables and rules cited above, and illustrated by one specific instance each, are

$$
\begin{aligned}
& F_{p}(0 ; 4,5) \\
= & \Gamma(1+c-e) \\
& \times\left(\frac{\Gamma(e-a-b) F_{n}(5 ; 0,3) \Gamma(1+a+b-e)}{\Gamma(s) \Gamma(-a+e) \Gamma(-b+e)}+\frac{\Gamma(a+b-e) F_{n}(3 ; 0,5) \Gamma(1+e-b-a)}{\Gamma(b) \Gamma(f-c) \Gamma(a)}\right)
\end{aligned}
$$


and

$$
\begin{aligned}
F_{p}(0 ; 4,5)= & \Gamma(1-f+b) \Gamma(1+c-f) \Gamma(1-f+a) \\
& \times\left(\frac{F_{n}(4 ; 0,2)}{\Gamma(s) \Gamma(f) \Gamma(1-f)}+\frac{F_{p}(5 ; 0,4)}{\Gamma(b) \Gamma(c) \Gamma(a)}\right) .
\end{aligned}
$$

Surprisingly, the third equation [3, Eq. 7.4.4(6)] cited above, once parsed in the same manner turns out to be equivalent to (C.7) with the change $F_{p}(4 ; 0,2)=F_{p}(4 ; 2,3)$ and is therefore not independent, although when expanded in its full glory, this is not evident.

Finally, it is noted that three more independent equations between the $F_{n}$ and $F_{p}$ functions can be found by changing the signs of the $r_{i}$ terms in [21, Eq. (13)]. This has the effect of redefining the parameters $\alpha_{l m n} \rightarrow 1-\alpha_{l m n}$ and $\beta_{m n} \rightarrow 2-\beta_{m n}$ in the various tables, as well as converting $F_{p}(u) \rightleftarrows F_{n}(u)$ and $s \rightarrow 1-s$. Thus (C.5) becomes

$$
\begin{aligned}
F_{n}(0)= & -\frac{\pi \Gamma(a)}{\sin (\pi(1+b-c))} \\
& \times\left(\frac{F_{p}(2)}{\Gamma(1-e+b) \Gamma(1-f+b) \Gamma(1-c)}-\frac{F_{p}(3)}{\Gamma(1+c-e) \Gamma(1+c-f) \Gamma(1-b)}\right),
\end{aligned}
$$

this time omitting the superfluous second and third parameters from $F_{p}(0), F_{p}(2)$ and $F_{p}(3)$, yet retaining all combinations of parameters in a form that a patient reader could identify as $\alpha_{l m n}$ or $\beta_{m n}$ from Luke's Tables. Similarly, (C.6) and (C.7) become

$$
\begin{aligned}
F_{n}(0)= & \frac{\pi \Gamma(e-c)}{\sin (\pi(1+e-b-a))} \\
& \times\left(\frac{F_{p}(5)}{\Gamma(1-e+b) \Gamma(1-e+a) \Gamma(1-s)}-\frac{F_{p}(3)}{\Gamma(1-b) \Gamma(1+c-f) \Gamma(1-a)}\right)
\end{aligned}
$$

and

$F_{n}(0)=\Gamma(-b+f) \Gamma(f-c) \Gamma(f-a)\left(-\frac{\sin (\pi f) F_{p}(4)}{\pi \Gamma(1-s)}+\frac{F_{n}(5)}{\Gamma(1-b) \Gamma(1-c) \Gamma(1-a)}\right)$.

A collection consisting of six such equations is sufficient to interrelate all possible three-term relations among $120{ }_{3} F_{2}(1)$ that can be identified as $F_{p}(u)$ and/or $F_{n}(u)$ [4, Section 4.3.2]. Luke [21, Eq. 3.13(26)] and Slater [4, Eq. (4.3.2.5)] go on to reproduce an example from Bailey that uses the above to relate $F_{n}(0), F_{p}(0)$ and $F_{p}(5)$ algebraically. Luke does not say which six equations he used. Similarly, at this same point, Slater refers to "the relation corresponding to [4, Eq. 4.3.2.1)] which connects $F_{p}(5), F_{n}(0)$ and $F_{n}(2) \ldots$ " but she never identifies the corresponding relation. Both references in Luke and Slater correspond to (C.5) here. It is reasonable, but not assured, to assume that Slater, Luke (and Bailey) based this example on the three wellestablished 3-part relations [3, Eqs. 7.4.4(3)-(5)] that gave rise to the above (Note: Bailey refers to Hardy and Whipple in a footnote at this point but also does not say 
which equations were used); Slater [4, Eq. (4.3.2.4)] identifies one. How are other relations found?

Dealing with the case of $F_{n}(1)$ and $F_{p}(1)$ (missing from all six of the above), note that $F_{n}(1 ; u, v)$ and $F_{n}(2 ; u, v)$ are related by an interchange of two of $a, b$ or $c$. For example, under the (symmetric) exchange $a \leftrightarrows b$, (C.5) becomes

$$
F_{p}(0)=\frac{\pi \Gamma(1-b)}{\sin (\pi(a-c))}\left(-\frac{F_{n}(1)}{\Gamma(-a+e) \Gamma(f-a) \Gamma(c)}+\frac{F_{n}(3)}{\Gamma(e-c) \Gamma(f-c) \Gamma(a)}\right)
$$

along with its complement

$$
\begin{aligned}
& F_{n}(0) \\
= & \frac{\pi \Gamma(b)}{\sin (\pi(a-c))}\left(\frac{F_{p}(1)}{\Gamma(1-e+a) \Gamma(1-f+a) \Gamma(1-c)}-\frac{F_{p}(3)}{\Gamma(1+c-e) \Gamma(1+c-f) \Gamma(1-a)}\right) .
\end{aligned}
$$

and this establishes that all the basic functions $F_{n}$ and $F_{p}$ are at least accessible from the independent relations. When written in labelled form (C.11) becomes

$$
F_{p}(0)=\frac{\pi \Gamma\left(\alpha_{013}\right)}{\sin \left(\pi \beta_{13}\right)}\left(\frac{F_{n}(1)}{\Gamma\left(\alpha_{234}\right) \Gamma\left(\alpha_{235}\right) \Gamma\left(\alpha_{345}\right)}-\frac{F_{n}(3)}{\Gamma\left(\alpha_{214}\right) \Gamma\left(\alpha_{215}\right) \Gamma\left(\alpha_{145}\right)} .\right)
$$

Notice that (C.4) and (C.13), related by the interchange $a \leftrightarrows b$, are equivalently related by the interchange of the numeric labels $1 \leftrightarrows 2$; this demonstrates the fundamental basis of the notation - the equality of the six equations chosen as a basis is invariant under interchange of numeric labels. With this understanding, all other relationships can be found. For example, consider the independently derived result (B.8)

$$
F_{p}(0)=\left(\frac{\sin (\pi e) F_{n}(2)}{\pi \Gamma(f-b)}+\frac{F_{p}(4)}{\Gamma(e-c) \Gamma(-a+e) \Gamma(b)}\right) \Gamma(1-c) \Gamma(1-a) \Gamma(1-e+b)
$$

along with its complement

$$
F_{n}(0)=\left(\frac{F_{n}(4)}{\Gamma(1+c-e) \Gamma(1+a-e) \Gamma(1-b)}-\frac{\sin (\pi e) F_{p}(2)}{\pi \Gamma(1-f+b)}\right) \Gamma(c) \Gamma(a) \Gamma(-b+e) .
$$

To demonstrate that (C.14) can be obtained from one (or more) of the six independent relations given above, start with (C.7), written in labelled form

$$
F_{p}(0)=\left(\frac{\sin \left(\pi \beta_{50}\right) F_{n}(4)}{\Gamma\left(\alpha_{123}\right) \pi}+\frac{F_{p}(5)}{\Gamma\left(\alpha_{245}\right) \Gamma\left(\alpha_{345}\right) \Gamma\left(\alpha_{145}\right)}\right) \Gamma\left(\alpha_{024}\right) \Gamma\left(\alpha_{014}\right) \Gamma\left(\alpha_{034}\right) .
$$

Interchange all labels $4 \leftrightarrows 2$ followed by the interchange $5 \leftrightarrows 4$ to find

$$
F_{p}(0)=\left(\frac{\sin \left(\pi \beta_{40}\right) F_{n}(2)}{\Gamma\left(\alpha_{153}\right) \pi}+\frac{F_{p}(4)}{\Gamma\left(\alpha_{524}\right) \Gamma\left(\alpha_{324}\right) \Gamma\left(\alpha_{124}\right)}\right) \Gamma\left(\alpha_{012}\right) \Gamma\left(\alpha_{052}\right) \Gamma\left(\alpha_{032}\right)
$$


which, when written in terms of the basic parameters, is identifiable as (C.14).

To derive (B.7) is more interesting. Written in this notation, (B.7) becomes

$$
\begin{aligned}
F_{p}(0)= & \frac{\sin (\pi e) \sin (\pi f) \Gamma(1-a) \Gamma(1-b) \Gamma(1-c)}{\sin (\pi(e-f))} \\
& \times\left(\frac{F_{p}(4)}{\sin (\pi e) \Gamma(-b+e) \Gamma(e-c) \Gamma(-a+e)}-\frac{F_{p}(5)}{\sin (\pi f) \Gamma(-b+f) \Gamma(f-c) \Gamma(f-a)}\right)
\end{aligned}
$$

and its derivation from any one of the six independent relations by interchanging labels becomes questionable, because none of them individually relate three $F_{p}$ functions. Choose (C.8) and (C.9) as a convenient staring point, equate the right-hand sides of both and solve for $F_{p}(2)$. Written in labelled notation, the solution is

$$
\begin{aligned}
F_{p}(2)= & \left(\frac{1}{\Gamma\left(\alpha_{035}\right)}+\frac{\Gamma\left(\alpha_{124}\right) \sin \left(\pi \beta_{23}\right)}{\Gamma\left(\alpha_{145}\right) \Gamma\left(\alpha_{023}\right) \sin \left(\pi \beta_{35}\right)}\right) \frac{\Gamma\left(\alpha_{012}\right) \Gamma\left(\alpha_{024}\right) \Gamma\left(\alpha_{025}\right)}{\Gamma\left(\alpha_{034}\right) \Gamma\left(\alpha_{013}\right)} F_{p}(3) \\
& -F_{p}(5) \frac{\Gamma\left(\alpha_{124}\right) \sin \left(\pi \beta_{23}\right) \Gamma\left(\alpha_{024}\right) \Gamma\left(\alpha_{012}\right)}{\Gamma\left(\alpha_{045}\right) \sin \left(\pi \beta_{35}\right) \Gamma\left(\alpha_{015}\right) \Gamma\left(\alpha_{145}\right)}
\end{aligned}
$$

Now perform the interchange of numeric labels $3 \leftrightarrows 4$, followed by the interchange $0 \leftrightarrows 2$, revert to the representation in terms of the underlying parameters, and after some simplification, (C.18) will be found.

In the section dealing with the generalization of Shpot and Srivastava's result (B.3), it was claimed that their [18, Eq.(31)] (see (D.11)) could easily be obtained from the "well known" result (B.5). A quick scan of [21, Table 3.5] shows that the two ${ }_{3} F_{2}(1)$ appearing in (B.5) can be identified as $F_{n}(2 ; 3,1)$ and $F_{n}(3 ; 1,2)$ as discussed above. Simple substitution into (C.5) will yield (B.5), justifying the remark that it is at least a "known", if not a "well known", result. In the case of Paris and Miller, their extended result (B.2) once parsed as discussed here can be written

$$
F_{p}(0)=\left(\frac{\sin (\pi e) F_{n}(3)}{\pi \Gamma(f-c)}+\frac{F_{p}(4)}{\Gamma(-b+e) \Gamma(-a+e) \Gamma(c)}\right) \Gamma(1+c-e) \Gamma(1-b) \Gamma(1-a)
$$

where specifically $F_{p}(0)=F_{p}(0 ; 4,5), F_{p}(4)=F_{p}(4 ; 1,2)$ and $F_{n}(3)=F_{n}(3 ; 1,2)$. To derive (C.20) from the above, solve for $F_{n}(2)$ in (C.5) and substitute into (C.14). So in the sense discussed here (C.20) is a known result.

\section{A collection of ${ }_{3} F_{2}(1),{ }_{4} F_{3}(1)$ and some lemmas}

The following is a collection of relevant results gathered from sources scattered throughout the literature, plus a few lemmas.

\section{- Minton Karlsson}

Minton [11] and Karlsson [12] show that, when a top parameter exceeds a bottom parameter by a positive integer $n$, the order of any ${ }_{p+1} F_{p}(1)$ can be reduced by 
one and replaced by a sum of $n$ terms. In the case $p=3$, this gives

$$
\begin{aligned}
& { }_{4} \mathrm{~F}_{3}(a, b, c, e+n ; e, f, g ; 1) \\
= & \frac{\Gamma(1+n) \Gamma(e) \Gamma(f) \Gamma(g)}{\Gamma(a) \Gamma(b) \Gamma(c)} \\
& \times \sum_{k=0}^{n} \frac{\Gamma(k+a) \Gamma(b+k) \Gamma(c+k){ }_{3} \mathrm{~F}_{2}(k+a, b+k, c+k ; f+k, g+k ; 1)}{\Gamma(k+1) \Gamma(1+n-k) \Gamma(e+k) \Gamma(f+k) \Gamma(g+k)} .
\end{aligned}
$$

See also [14].

\section{- Sheppard-Andersen Theorem}

Based on [24, Corollary 3.3.4], the general result for a k-balanced, terminating ${ }_{3} F_{2}(1)$ is given by

$$
\begin{aligned}
& { }_{3} \mathrm{~F}_{2}(a, b,-n ; c, k-n+a+b-c ; 1) \\
= & -\frac{\pi(-1)^{n} \Gamma(a-c+1)(-1)^{k} \Gamma(k-n+a+b-c) \Gamma(n+1) \Gamma(k) \Gamma(c)}{\sin (\pi(c-b)) \Gamma(k-n+b-c) \Gamma(k+b-c+a) \Gamma(n+c) \Gamma(a)} \\
& \times \sum_{j=0}^{N} \frac{\Gamma(a+j)}{\Gamma(1+n-j) \Gamma(-b+c-k+1+j) \Gamma(a-n-c+1+j) \Gamma(1+j) \Gamma(k-j)}
\end{aligned}
$$

where $N=\min (k-1, n)$. In the case $k=2$ a simpler result [25, Eq. (2.9), misprinted] is

$$
\begin{aligned}
& { }_{3} \mathrm{~F}_{2}(a, b,-n ; c, 2-n+a+b-c ; 1) \\
= & \frac{\Gamma(-b+c+n-1) \Gamma(n+c-a) \Gamma(c-a-b-1) \Gamma(c)}{\Gamma(c-b-1) \Gamma(c-a) \Gamma(-b+c+n-1-a) \Gamma(n+c)}\left(1-\frac{n a}{(c-b-1)(-a+n+c-1)}\right)
\end{aligned}
$$

REMARK. The result (D.2) is usually referenced in the literature to an inaccessible paper by Sheppard [26], where it is also usually noted that Andersen [27] obtained (D.3) independently. In fact, Andersen obtained the following result (transcribed in hypergeometric notation)

$$
\begin{aligned}
& { }_{3} \mathrm{~F}_{2}(1, a, m+b ; 2+m, a+b ; 1) \\
= & \frac{(-1)^{m} \sin (\pi a) \sin (\pi b) \Gamma(1-b-m) \Gamma(b+a) \Gamma(-a+1) \Gamma(2+m)}{(-m+a-1) \pi^{2}(b-1)} \\
& -\frac{(1+m)(b+a-1)}{(-m+a-1)(b-1)}
\end{aligned}
$$

and a similar result for the case where the bottom parameter is $a+b+1$, both of which are special cases of [17, Entry 28]. 


\section{- Whipples tranformation}

The result (D.2) is based upon the following transformation of a terminating 1balanced ${ }_{4} F_{3}(1)$ due to Whipple (1926) (see [24, Theorem 3.3.3])

$$
\begin{aligned}
& { }_{4} \mathrm{~F}_{3}(a, b, c,-n ; d, e, f ; 1) \\
= & \frac{\Gamma(n+e-a) \Gamma(n+f-a) \Gamma(e) \Gamma(f){ }_{4} \mathrm{~F}_{3}(a,-n, d-b, d-c ; d, a+1-n-f, a+1-n-e ; 1)}{\Gamma(e-a) \Gamma(f-a) \Gamma(n+e) \Gamma(n+f)}
\end{aligned}
$$

where $f=a+b+c+1-d-e-n$.

Suppose $d=c-1$. Then (D.5) gives the following result for a special (MintonKarlsson), 1-balanced, terminating ${ }_{4} F_{3}(1)$

$$
\begin{aligned}
& { }_{4} \mathrm{~F}_{3}(-n, a, b, c ; c-1, e, a+b+2-n-e ; 1) \\
= & A \frac{\Gamma(a+b+2-n-e) \Gamma(n+e-a-1) \Gamma(b+1-e) \Gamma(e)}{\Gamma(b+2-n-e) \Gamma(a+b+2-e) \Gamma(n+e) \Gamma(e-a)}
\end{aligned}
$$

where

$$
A=-e^{2}+(a+b-n+2) e-\frac{(b+1)(a-c+1)(n-1)}{c-1}+\frac{(-b-1+n) a c}{c-1} .
$$

Other variations are apparent.

- A special ${ }_{q+1} \mathbf{F}_{q}(1)$

Prudnikov et. al. [3, Eq. 7.10.2(6)] give the following general result ${ }_{q+1} \mathrm{~F}_{q}(a, b, \ldots, b ; b+1, \ldots, b+1 ; 1)=\frac{(-1)^{q-1} b^{q} \Gamma(1-a)}{(q-1) !} \frac{\partial^{q-1}}{\partial b^{q-1}}\left(\frac{\Gamma(b)}{\Gamma(1+b-a)}\right)$.

When $q=4$ and $b=1$, we find

$$
\begin{aligned}
& (1-a){ }_{5} \mathrm{~F}_{4}(1,1,1,1, a ; 2,2,2,2 ; 1) \\
= & 1 / 6(\Psi(2-a)+\gamma)^{3} \\
+ & \left(1 / 12 \pi^{2}-1 / 2 \Psi^{\prime}(2-a)\right)(\Psi(2-a)+\gamma)+1 / 3 \zeta(3)+1 / 6 \Psi^{(2)}(2-a)
\end{aligned}
$$

and similarly with $q=5$,

$$
\begin{aligned}
& (1-a){ }_{6} \mathrm{~F}_{5}(1,1,1,1,1, a ; 2,2,2,2,2 ; 1) \\
= & 1 / 8 \Psi^{\prime}(2-a)^{2}+\frac{\pi^{4}}{160}-1 / 24 \Psi^{(3)}(2-a) \\
& +1 / 24(\Psi(2-a)+\gamma)^{4}+\left(1 / 3 \zeta(3)+1 / 6 \Psi^{(2)}(2-a)\right)(\Psi(2-a)+\gamma) \\
& -\left(1 / 4(\Psi(2-a)+\gamma)^{2}+1 / 24 \pi^{2}\right) \Psi^{\prime}(2-a)+1 / 24 \pi^{2}(\Psi(2-a)+\gamma)^{2} .
\end{aligned}
$$




\section{- A result from Shpot and Srivastava [18]}

The following reproduces [18, Eq. (31)]

$$
\frac{{ }_{3} \mathrm{~F}_{2}(a, b, c ; b+1+m, c+1+n ; 1) \Gamma(b) \Gamma(c)}{\Gamma(b+1+m) \Gamma(c+1+n)}=T(a, b, c, m, n)+T(a, c, b, n, m)
$$

where

$$
T(a, b, c, m, n)=\frac{\Gamma(b) \Gamma(1-a) \Gamma(c-b){ }_{3} \mathrm{~F}_{2}(b,-m, b-c-n ; 1+b-a, 1+b-c ; 1)}{\Gamma(1+b-a) \Gamma(n+1+c-b) \Gamma(m+1)}
$$

- From a previous work [28, Theorem 2.2]

$$
\begin{aligned}
& { }_{4} \mathrm{~F}_{3}(1,1,1,1 ; 2,2, d ; 1) \\
= & (d-1)\left(\Psi^{(2)}(d-1) / 2-\left(\Psi^{\prime}(d-1)+\pi^{2} / 6\right)(\gamma+\Psi(d-1))\right. \\
& \left.+2 \zeta(3)-\frac{1}{\Gamma(2-d)} \sum_{k=0}^{\infty} \frac{\Psi^{\prime}(k+2) \Gamma(3-d+k)}{\Gamma(1+k)(1+k)^{2}}\right)
\end{aligned}
$$

\section{- Lemma 1}

Because

$\sum_{k=0}^{n-1} \frac{\Gamma(n+k-b)(-1)^{k}}{\Gamma(k+1)^{2} \Gamma(n-k)}=\frac{\Gamma(n-b){ }_{2} \mathrm{~F}_{1}(n-b,-n+1 ; 1 ; 1)}{\Gamma(n)}=-\frac{(-1)^{n} \Gamma(n-b)^{2}}{\Gamma(-b+1) \Gamma(n)^{2}}$,

differentiate with respect to $b$ giving

$$
\sum_{k=0}^{n-1} \frac{\Gamma(n+k-b)(-1)^{k} \Psi(n+k-b)}{\Gamma(k+1)^{2} \Gamma(n-k)}=-\frac{(-1)^{n} \pi(-\Psi(1-b)+2 \Psi(n-b)) \Gamma(b)}{\sin (\pi b) \Gamma(n)^{2} \Gamma(-n+b+1)^{2}},
$$

\section{- Lemma 2}

From [17, Entry 13], corresponding to ${ }_{3} \mathrm{~F}_{2}(1,1, a ; 1+a, 1+a ; 1)$, a case contiguous to Whipple's theorem we have

$$
\sum_{k=1}^{\infty} \frac{\Gamma(k)}{(k+a-1) \Gamma(k+a)}=\frac{\Psi^{\prime}(a / 2)-\Psi^{\prime}(a / 2+1 / 2)}{2 \Gamma(a)},
$$

and, after differentiating with respect to $a$, we obtain

$$
\begin{aligned}
& \sum_{k=1}^{\infty} \frac{\Gamma(k) \Psi(k+a)}{(k+a-1) \Gamma(k+a)} \\
= & -\sum_{k=1}^{\infty} \frac{\Gamma(k)}{(k+a-1)^{2} \Gamma(k+a)} \\
+ & \frac{\left(\Psi^{\prime}(a / 2)-\Psi^{\prime}(a / 2+1 / 2)\right) \Psi(a) / 2-1 / 4 \Psi^{(2)}(a / 2)+1 / 4 \Psi^{(2)}(a / 2+1 / 2)}{\Gamma(a)}
\end{aligned}
$$




\section{- Lemma 3}

By adding and subtracting a term corresponding to $k=-1$, from its series representation, we have

$$
{ }_{4} \mathrm{~F}_{3}(1,2,2-a, a+1 ; 3,3,3 ; 1)=\frac{8 \pi{ }_{4} \mathrm{~F}_{3}(1,1, a, 1-a ; 2,2,2 ; 1)}{(1-a) \Gamma(1-a) \Gamma(a) a \sin (\pi a)}-\frac{8}{(1-a) a}
$$

so that, after the invocation of (??)

$$
\begin{aligned}
& { }_{4} \mathrm{~F}_{3}(1,2,2-a, a+1 ; 3,3,3 ; 1) \\
= & \frac{\sin (\pi a)}{a^{2}(a-1)^{2} \pi}\left(16 \Psi^{\prime}(a)-8 \Psi^{\prime}(a / 2)+8 \frac{a^{2}-a+1}{a^{2}(a-1)^{2}}\right) \\
& -16 \frac{\Psi(a+1)}{a^{2}(a-1)^{2}}-16 \frac{\gamma}{a^{2}(a-1)^{2}}+8 \frac{a^{4}-2 a^{3}+a^{2}+2 a-1}{a^{3}(a-1)^{3}} .
\end{aligned}
$$

\section{- Lemma 4}

From [17, Entry 26] after evaluating some limits,

$$
\begin{aligned}
{ }_{3} \mathrm{~F}_{2}(1,1, n ; n+1, n+1 ; 1) \\
=-\left(\sum_{k=0}^{n-3} \frac{(-1)^{k} \Psi(1+k)}{\Gamma(n-k-1)(n-k-1)^{2} \Gamma(1+k)}+\sum_{k=0}^{n-3} \frac{(-1)^{k}}{\Gamma(n-k-1)(n-k-1)^{3} \Gamma(1+k)}\right) n^{2} \Gamma(n) \\
+(-1)^{n}\left(1 / 2-(\Psi(n)+1) n^{3}+n^{2}\left(\gamma^{2} / 2+2 \gamma \Psi(n)+\pi^{2} / 12+3 / 2 \Psi(n)^{2}\right.\right. \\
\left.\left.+\Psi(n)-2 \Psi^{\prime}(n)+1 / 2 \Psi^{\prime}(n+1)+2\right)\right)
\end{aligned}
$$

\section{- Lemma 5}

From [17, Entry 13] - contiguous to Whipples theorem, and a generalization of (D.20)

$$
{ }_{3} \mathrm{~F}_{2}(1,1, a ; a+1, a+1 ; 1)=\frac{a^{2}}{4} \Psi^{\prime}(a / 2+1)+\frac{a^{2}}{4} \Psi^{\prime}(a / 2)-\frac{a^{2}}{2} \Psi^{\prime}(a / 2+1 / 2)+1
$$

so that, after differentiating we obtain

$$
\begin{aligned}
& \sum_{k=1}^{\infty} \frac{\Gamma(k) \Psi(k+a)}{(k+a-1) \Gamma(k+a)} \\
= & -\sum_{k=1}^{\infty} \frac{\Gamma(k)}{(k+a-1)^{3} \Gamma(k+a-1)} \\
+ & \frac{2 \Psi^{\prime}(a / 2) \Psi(a)-2 \Psi^{\prime}(a / 2+1 / 2) \Psi(a)-\Psi^{(2)}(a / 2)+\Psi^{(2)}(a / 2+1 / 2)}{4 \Gamma(a)}
\end{aligned}
$$

For a comparison of (D.20) and (D.21), see (??). 
- Lemma 6

Trivially,

$$
{ }_{3} \mathrm{~F}_{2}(1,1,2-a ; 2,2 ; 1)=\frac{\Psi(a)+\gamma}{\Gamma(a)}
$$

leading to

$$
\sum_{k=1}^{\infty} \frac{(-1)^{k} \Psi(a-k)}{k^{2} \Gamma(k) \Gamma(a-k)}=\frac{\Psi^{\prime}(a)}{\Gamma(a)}-\frac{\Psi(a)^{2}}{\Gamma(a)}-\gamma \frac{\Psi(a)}{\Gamma(a)}
$$

